\title{
HBIM TO VR. SEMANTIC AWARENESS AND DATA ENRICHMENT INTEROPERABILITY FOR PARAMETRIC LIBRARIES OF HISTORICAL ARCHITECTURE
}

\author{
R. Quattrini ${ }^{\text {a }}$, C. Battini ${ }^{\text {b }}$, R. Mammoli ${ }^{\text {a, }}$ \\ ${ }^{a}$ DICEA, Dept. of Civil and Building Engineering and Architecture, Polytechnic University of Marche, Ancona, Italy \\ r.quattrini@univpm.it,r.mammoli@pm.univpm.it \\ ${ }^{\mathrm{b}}$ DICCA, Dept. of Civil, Chemical and Environmental Engineering, University of Genova, Genova, Italy \\ carlo.battini@unige.it
}

Commission II, WG II/8

KEY WORDS: Semantic Modeling, Parametric Intelligence, Data Enrichment, Aware 3D Database, Visualization

\begin{abstract}
:
Recently we assist to an increasing availability of HBIM models rich in geometric and informative terms. Instead, there is still a lack of researches implementing dedicated libraries, based on parametric intelligence and semantically aware, related to the architectural heritage. Additional challenges became from their portability in non-desktop environment (such as VR). The research article demonstrates the validity of a workflow applied to the architectural heritage, which starting from the semantic modeling reaches the visualization in a virtual reality environment, passing through the necessary phases of export, data migration and management. The three-dimensional modeling of the classical Doric order takes place in the BIM work environment and is configured as a necessary starting point for the implementation of data, parametric intelligences and definition of ontologies that exclusively qualify the model. The study also enables an effective method for data migration from the BIM model to databases integrated into VR technologies for $\mathrm{AH}$. Furthermore, the process intends to propose a methodology, applicable in a return path, suited to the achievement of an appropriate data enrichment of each model and to the possibility of interaction in VR environment with the model.
\end{abstract}

\section{INTRODUCTION}

In recent years, several researches allowed the creation of a database incorporating Building Information Modeling (BIM) objects suitable in the historical urban context or buildings. The obtained models are at the same time, complete in dimensional - geometrical terms and rich in descriptive information, but the knowledge-based parametric intelligence is still lacking. The basic libraries in BIM software are increasingly updated and useful in the application on new projects, but in the case of historical heritage or restoration of historical buildings, the necessary elements are often unavailable or not appropriate to rules identified in the Treatises. Therefore, there is a strong need to create, in a BIM work environment, a series of objects with complex and irregular shapes, which can be implemented and managed through parametric data and with graphic and non-graphical information.There are various methods for data enrichment, able to be managed by practitioners in Historical Building Information Modeling (HBIM) environment, but there are limits when data processing is handled through other applications and 3D object are migrated out of modeling space. Actually, many problems arise from interoperability with nondesktop environments such as Augmented Reality (AR) and Virtual Reality (VR). The parametric model obtained in HBIM environment, appropriately enriched with qualitative data, during the export phase on other platforms, loses essential information that does not allow complete semantic comprehension and hierarchic structure of the 3D object.

\section{STATE OF ART}

Since its first definition (Murphy, McGovern and Pavia, 2009) HBIM is intended as a complex model composed by a library of parametric objects: those approach deals with systems capable of mapping smart objects to survey data, like point clouds or images in a whole informative management tool, reusing as much is possible already modeled families.

In architectural heritage representation or in virtual reconstruction of no more existing architecture the chance offered by the HBIM is to emphasize the use of a semantic construction of the digital model and to show conceptual similarity between the treaties and BIM (Apollonio, Gaiani and Sun, 2013). In this light, good advances are observed by new and robust solutions from $2 \mathrm{D}$ drawings for $\mathrm{CH}$ artifacts to the $3 \mathrm{D}$ modeling, able to allow the verification of the assumptions used during the reconstruction (Apollonio and Giovannini, 2015). The conceptual framework of this kind of application is showed in (De Luca, 2014): based on semantics, it is possible to structure $3 \mathrm{D}$ models allowing the use of a controlled vocabulary for each elements of the building, to identify and regroup elements in a coherent unit and to choice a representation type able to extract from shapes the necessary information.

The diffusion of BIM procedures in heritage documentation allowed to test and validate different specifications and quality about the Level of Development (LOD), Information (LOI) and Geometry (LOG). In these works it is possible to obtain reliable low cost 3D textured models that could be used in BIM platforms to create semantic-aware objects (Santagati and Lo Turco, 2016). In general, literature agrees that BIM systems have much improved thanks to the technological evolution and nowadays provide more refined tools to manage the processes related with architectural design and heritage representation (Bianchini et al., 2016). Other significant results are observed in management and restoration of built heritage, allowing a wide range of solution for the $3 \mathrm{D}$ modeling, thus adapting to new requirements (López et al., 2017) or in order to translate real shapes into "smart" digital architectural components organized into proper hierarchical BIM frameworks (Garagnani, 2015).

The UNI committee consolidated the LOD\& LOG definitions at the Italian level and working groups are scaling up this 
framework with an European extension. We assisted to a new subdivision of the LOD range (COMMITTE, 2017): from A to E for architectural design and LOD scale (F-G) for as built representation and restoration. This kind of publications can certainly contribute to widespread good practices from the research field to the professional one.

Interoperability issues are often investigated for the portability in other desktop environments, for example from BIM to FEM (Bassier et al., 2016) and MEP simulation, in which important practical results along the design process are obtained (Biagini et al., 2016), thanks to a high level of accuracy in data restitution. The present paper deals, instead, with the portability from BIM to VR: a quite novel topic. Regarding the migration from BIM environment to AR applications, the research approach is mainly conceived to adopt a design science enabling a mobile BIM system and to test it from the effectiveness and usability point of view (Chu, Matthews and Love, 2018). A theoretical analysis about perceiving and drawing through $\mathrm{AR}$ tools and a technical discussion about obstacles and potentials is provided in (Clini et al., 2016).

Other studies related with issue of migrating reliable data from survey and reality-based models to VR/AR applications are (Battini, Sorge 2016)(Battini and Vecchiattini, 2017). In these cases, authors demonstrated that Virtual Reality technology can be a valuable tool that allows carrying out different cultural tasks, from conservation to exploitation. VR could also be used as a more immediate and effective didactic tool. Other present visualization pipeline developed to exploit and access large and complex heritage 3D contents in an easy and interactive way through VR methodologies. In particular strong points arise for 3D models with a real-time fluent interaction (Jiménez Fernández-Palacios, Morabito and Remondino, 2017).

Currently moreover, there are some studies that demonstrate the ability to implement three-dimensional polygonal models from BIM platforms for use in VR environments (Liu et al., 2016). 3D models can help to understand and analyze data and game engine, such as Unity, are powerful to do this (Handel $e t$ al., 2009). Furthermore, Unity can be explored to produce a virtual environment for visual display and 3D files can be imported and exported (Dalton et al., 2018). These models are materially perfect but in the same way they lose quality of BIM environment, such as the possibility of finding the parametric information by which the geometries are made.

\section{METHODOLOGY}

The paper presents a novel method for migrating parametric intelligence and semantic structuring from the HBIM environment to visualization tools, often used in gaming and virtual reality interaction. The workflow starts with a modeling phase useful to define geometrical constraints and all related information. A first mandatory step is the formal structuring of the object (Figure 1): in our case study, the taxonomy follows the division of the order into its constituent elements (cyma, scotia, ovolo, etc.)

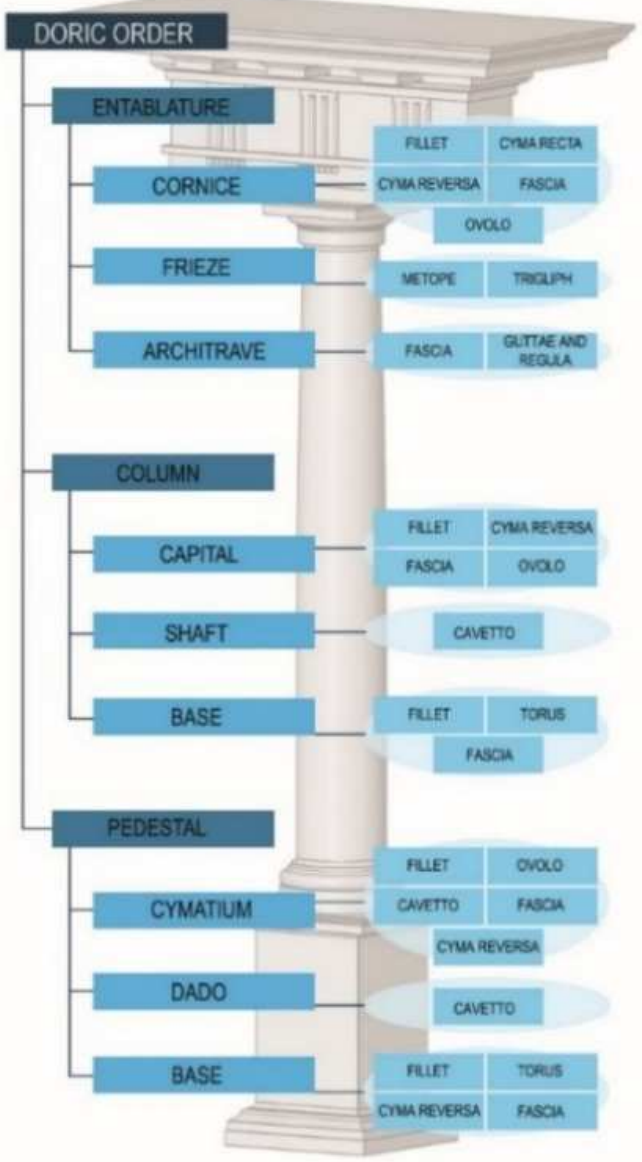

Figure 1. Formal structuring of Doric Order, taxonomy follows division of the Order in its moldings

This step provides also conservation of parent-child structure and rules, even though the format changes enabling interoperability with game engine. The second step is devoted to query the database and to verify the semantic awareness of all 3D objects and third step regards the visualization of the enriched model in non - desktop environment.

\subsection{Semantic Modeling}

The case study here presented is the development of a family of a Doric order, semantically aware and incapsulating its rules. We have chosen to work with it as it represents an easily identifiable and replicable model in historical architectures. The analysis of the order started with the study of the Treatises, specifically the Vitruvian one (Gros, 1997), and then we decided to focus on the geometric shapes and relations among the module and the moldings construct based on the module itself. These relations represent the central part of the parametric modeling phase and are able to describe completely the order.

Dealing with the parameterization of Doric order, we carried out a family composed by entablature, column and base, trough Autodesk Revit software. Observing the available families, it is clear that the morphology and the parameterization do not allow the maintenance of the proportions. Furthermore the software libraries disposition outlines how much the importance of .rfa category, linked to historic buildings, is still underestimated. They are in fact displaced in "Special Equipment" folder, in which there are different elements categories. There is also a "Classical 
colums" folder with some families, but they are not suitable to rules of treatises.

The geometry of the surfaces and volumes is sometimes irregular and complex, especially regarding moldings like: torus, cyma reversa, cavetto, etc. So, for the modeling phase is mandatory to study the geometrical rules that set proportions and relations through parts (

Figure 2). The relations between Modulo and components of the Order are basically expressed by mathematical formulas that allow a general connection of single parts.

After this first phase we start to create new family type using family types tool, exploiting existing software instruments to set different parameters and values according to the characteristic of moldings.

This procedure enables the implementation of parametric intelligence based on the knowledge of architectural heritage starting from commercial software without a computer scientist expertise.

For pedestal.rfa, for example, we create an instance parameter for any moldings, selecting a discipline (common), a type (length) and a group (dimensions) to correctly display the parameter under properties palette.
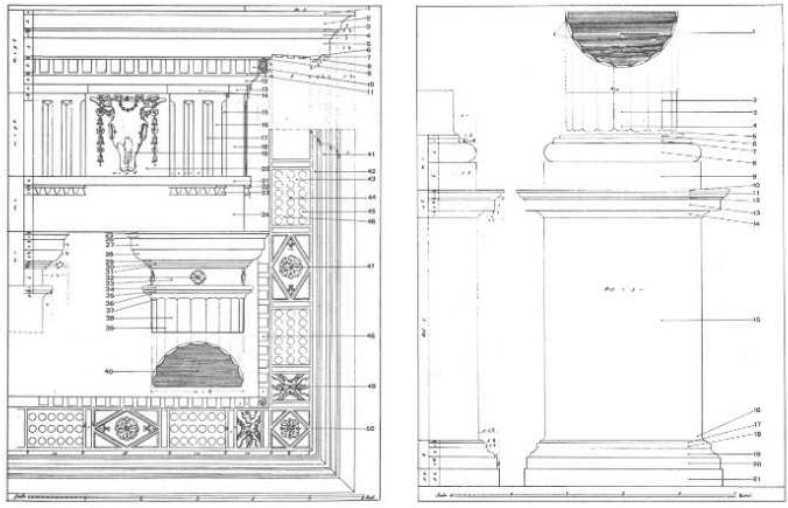

Figure 2. Entablature, column and pedestal in treatise. The images are from the text "Le membra degli ornamenti" (Morolli, 1986)

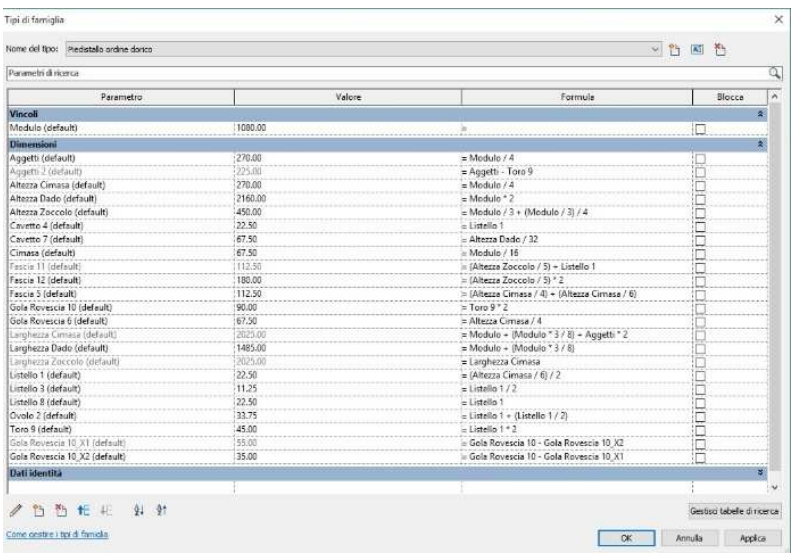

Figure 3. Creation of an instance parameter: Family type, parameter name, value and formula

As it is in all orders, the Modulo controls all the parts that describe the geometric dimensions, so once all instance parameters are defined, we relate them to it, using formulas that we have previously check (Figure 3 ). Instance parameters and its formulas allow the geometric profile, composed by moldings, to work correctly in case of scale change or modulo adjustments.

Regarding the profile and its extrusion, we try two different ways: both have first part in common (beforehand described). Our first attempt was to draw on 2D work plane using reference lines and planes and extruding every single molding fixing a work plane and two constraints: start and end. These constraints are all editable from properties box and is possible to gather each one to a family parameter (choosing from the list that we previously made).

The second attempt was to extrude a single profile that represented the entire pedestal.rfa family.
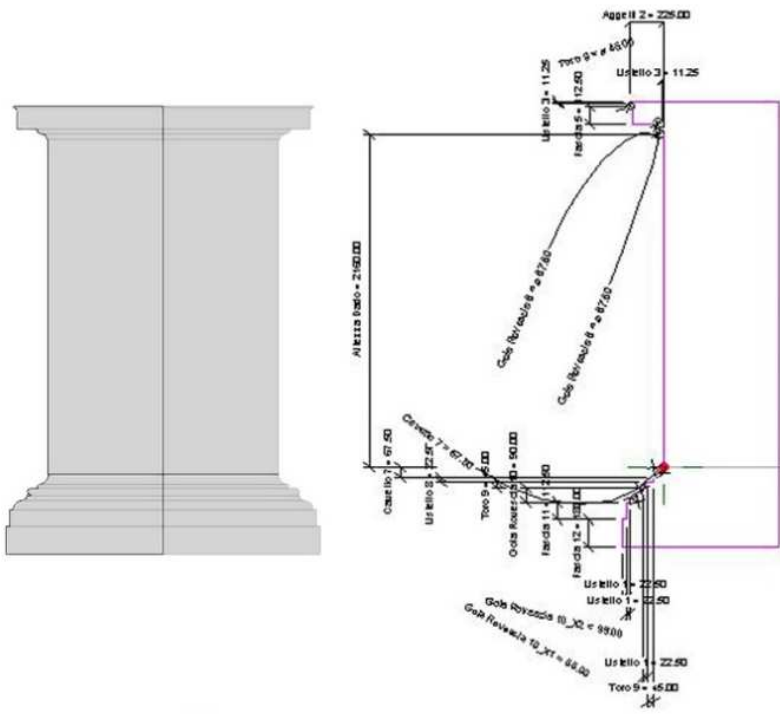

Figure 4. Pedestal profile (4A. Geometrical outline, 4B. Tags - Instance parameters)

We started from the definition of path and geometrical outline (Figure 4A) and then we used measure system to relate each element to instance parameters (Figure 4B). To do this we assigned tags to measures, knowing its importance and meaning in building information modelling environment. Tags are more than simple text box, in fact, even if they are annotation objects we can modify and customize them like loadable families or instance parameters. This kind of approach works better than previous one, in fact when we tried to change the value of Modulo all the forming parts of pedestal change in terms of proportions. In the previous one, instead, some artefacts occur.

Both attempts show that in this approach there are some weak points, that could be undoubtedly future basis for further reflections and studies on this topic, and some strong ones.

All the geometrical relations and formulas are defined, but this implementation requires an in - depth knowledge of classical orders and its translation into rules in a no automatic manner. This method does not provide any kind of automation in its first phase, so it represents certainly a weak point because for each molding we need to create one or more instance parameter.

This methodology has also been applied to the construction of column and entablature families (Figure 5)

Another solution for adding same rules concerns the determination of the constraints according to different modalities: application of equivalent dimensions (EQ) and parameterization from properties panel with insertion of mathematical formulas. In this case we use equivalent dimensions with reference planes to fix proportions when 
heights vary, while in the case of parameterization using formulas, we use them as constraints related to the module. This way, that seems a little bit fast and clear than previous one, was discarded because it does not allow further steps regarding data migration through Dynamo due to nested families.
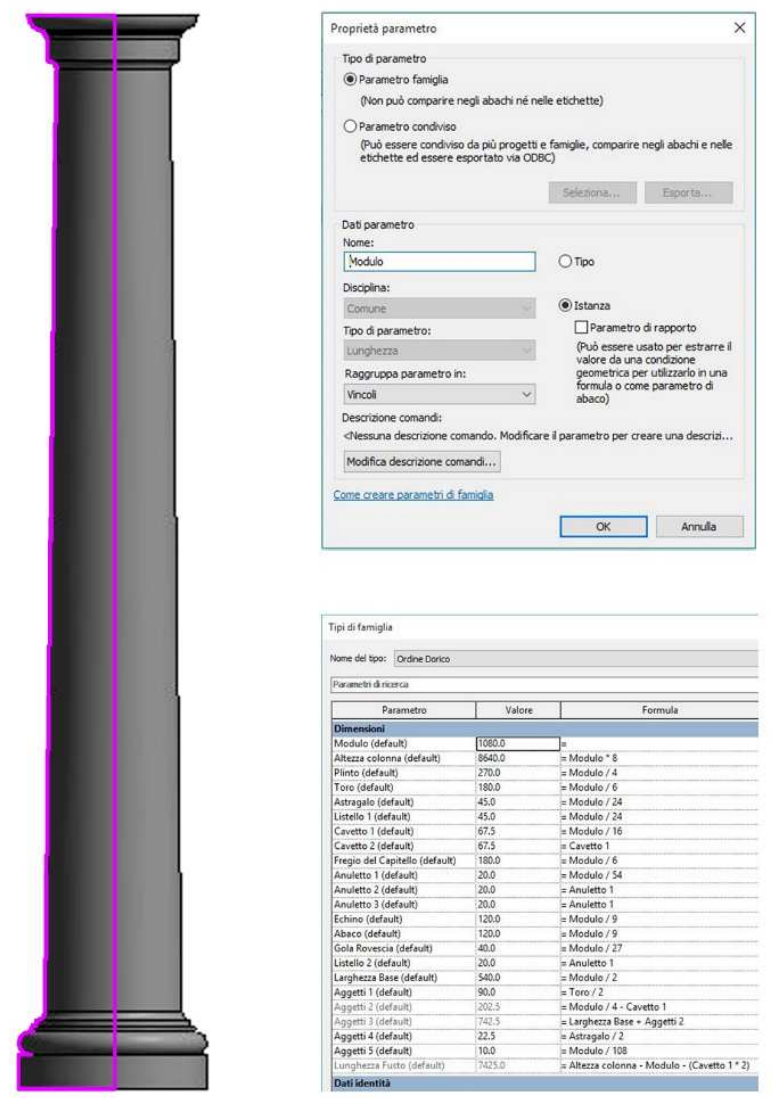

Figure 5. Revit 3D model of Doric Column, parameters properties and family type with mathematical formulas

\subsection{Data migration and management}

New advances in this field allow a portable and interoperable conceptualization for the knowledge of architectural heritage. This phase regards information, geometries, constraints, equivalent dimensions and formulas that can become parameters for the construction of our model. How this data can be used in three-dimensional modeling software and interactive visualization?

The proposed experimentation involves the study of a workflow for the transposition of BIM model in VR viewer, making it possible visualization and parametric information editing. Realized models are materially perfect but they lose quality of BIM environment.

This lack is mainly due to the difficulty in converting from BIM database to another type capable of interacting with other software solutions. Moreover, the absence of implemented information is given by the essential need to convert a parametric model, able to update itself to modify its parameters, in a static polygonal one, defined spatially by vertices, edges and polygons.

The proposed workflow involves the creation of a semiautomatic system capable of transferring parametric information into a MySQL database and then to access it through a dynamic viewer. To achieve this goal, it has been necessary to use a multiple systems of programming environments for both the initial phase of extraction data and the creation of the environment in the mobile display/management software.

A mandatory step was to understand how the parametric information is managed by the BIM Revit software.

For this purpose, we used the additional plugin Dynamo, open source visual programming environment that allows to compose custom algorithms using nodes and relationships. The enriched model can be subdivided and interrogated in all its components and it is possible to extract the necessary information to create a database that can also be queried by other software. In this case study Dynamo was used for exporting geometric properties of families, such as dimensions and mathematical formulas. Each node has a function - from the definition of parameters to the design of geometries - and maintains its link with preceding ones, in a flow that closely looks like programming. Dynamo is, in fact, used mainly for the creation of complex geometries, based on mathematical formulas, which can be modified at any time. At the same time Dynamo provides multiple nodes capable of managing data and interacting with the system parameters: extraction of information, composition of strings for communication with the MySQL database, recovery and updating of parametric values from the MySQL database.

During data extraction phase, the script we made provides that it is possible to recover the information of the selected instance including the properties of the family type after we have selected a model in the Revit project where the .rfa families were previously inserted.

The software gives back this information, in a random manner and in a list form: each index has a match to a field and its value. The specificity of this first list is the heterogeneous presence of all the information, not grouped by type. For the proposed effort in this experimentation, we decide to select only the data belonging to the group "dimension" and "identity data". Working with particular nodes, the workflow continued with the creation of two lists: names of the fields and their values. A follow up was done to determinate mathematical formulas inserted in phase of construction of the family. These were extracted and, if any, they were replaced to the list of values in order to insert them later in MySQL database.

In the "dimension" group there are also the parameters "Area" and "Volume" that have been deleted from the list, because during the analysis of the presence of mathematical formulas, they created problems with the elimination of some fields and with the misalignment of lists.

Following operations concerned the creation of textual strings in SQL format for the creation and population of the database. In this phase it was essential to define the structure of the DB with the creation of the necessary tables to gather and link the information collected. To guarantee the correspondence between model properties and layout of a BIM project, the structure of the DB requires the creation of: a) a table that collects the names of the Revit projects analyzed; b) a link table with the name of the project and the list of the elements, c) the relative family types and the connection with the threedimensional model; d) specific tables for each type of family where the names of columns are names of parameters (Figure 6) 


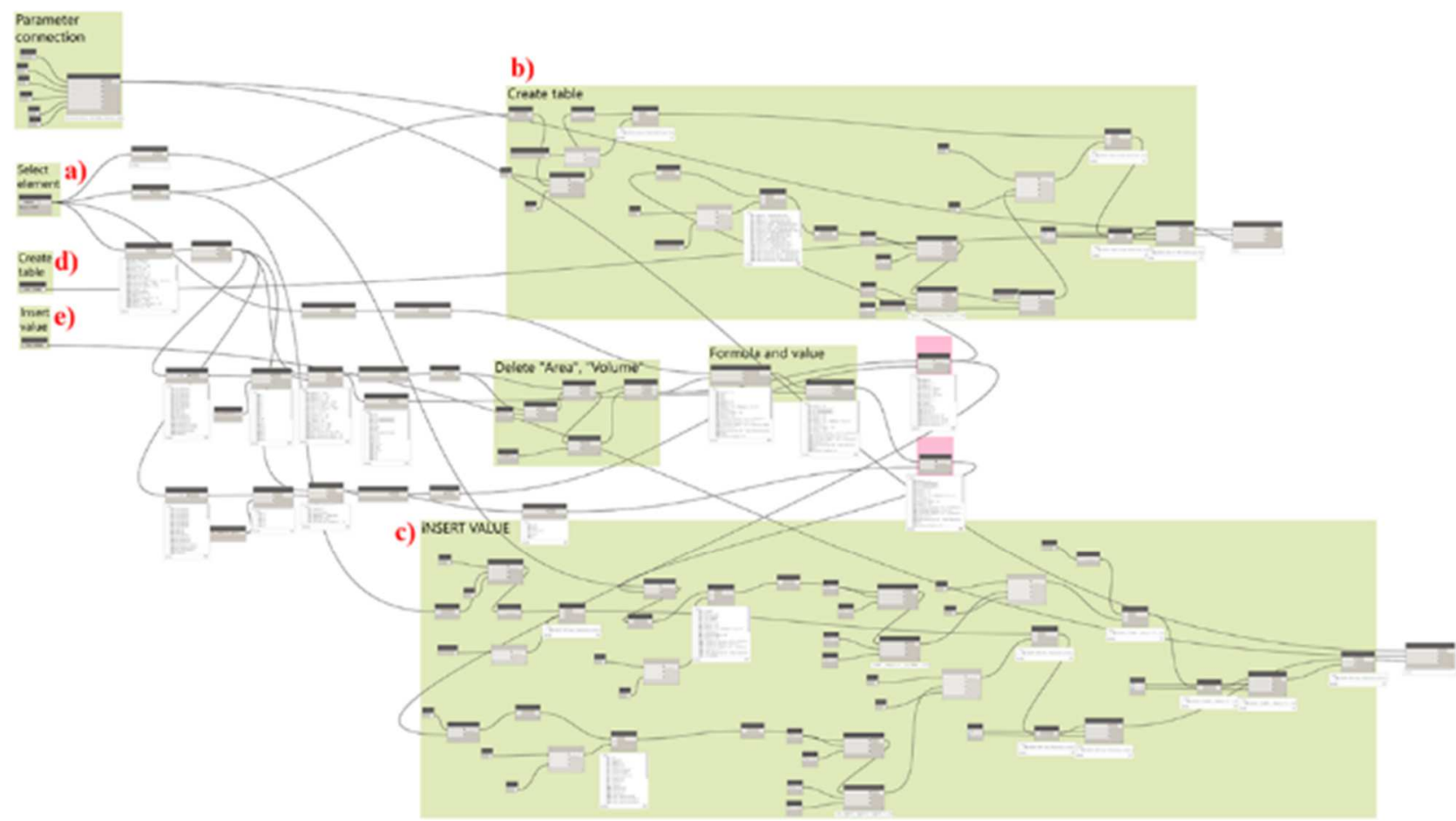

Figure 6.Workflow sequence for creating the type family table and entering values for the selected element: a) selection element; b) Generation of MySQL string for creating the table; c) Generation of MySQL string for entering values; d) and e) keys for running the script.

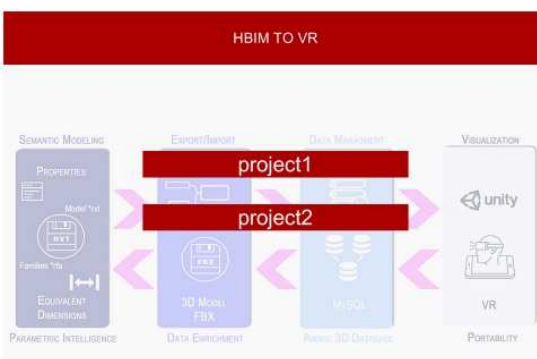

a)
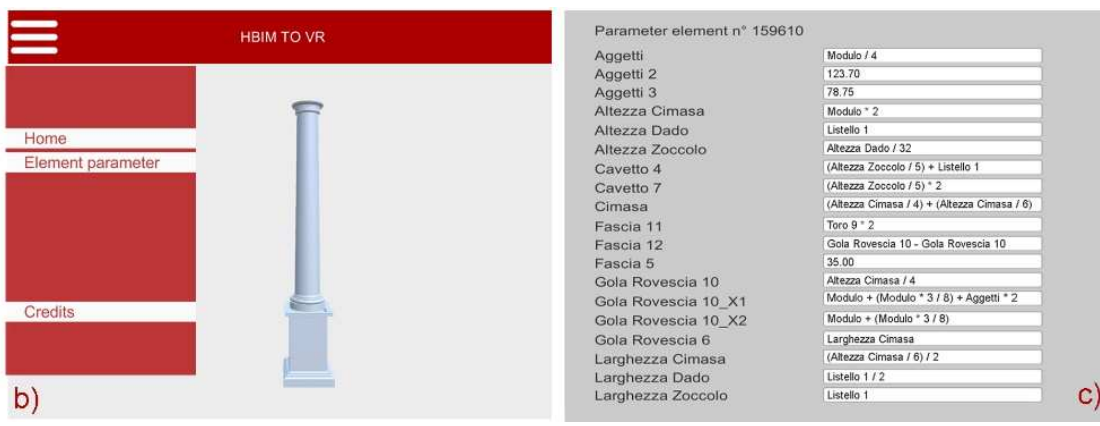

Figure 7. Some screenshots of the application developed: a) list of Revit projects on the server side; b) VR view of project elements and popup menu with programmed features; c) parameters of the selected element.

\subsection{Visualization}

Last step concerns the creation of VR app once created and populated tables, for a mobile viewer. The application was created within the Unity 3D multi-platform software that integrates powerful graphic engine for the visualization of three-dimensional models and use of programming languages for interaction with the virtual environment.

The proposed application provides that it can connect to the server and retrieve both the information contained within the DB and the three-dimensional models. All implemented actions were made using $\mathrm{C \#}$ programming language.

The proposed application provides that it can connect to the server and retrieve both the information contained within the DB and the three-dimensional models. All implemented actions were made using $\mathrm{C \#}$ programming language. Application framework includes the creation of two scenes: the first (Menu) dedicated to recovering the inserted Revit projects; the second (Home) that is reserved for viewing and interaction with the instances loaded on the server dedicated to each specific project.
The connections between client and server are guaranteed using the PHP language.

When the application is launched, the first screen that appears is "Menu" which restores the list of projects automatically connecting to the internet. The generation of list is dynamic, allowing constant updating of information. Clicking on a specific project we can automatically accesses to "Home" scene. During the transition between scenes, the application queries DB and recovers the list of items included from the specific project table, even providing the download of related 3D models. In "Home" scene the user can navigate in threedimensional space using zoom and rotation commands.

Layout includes a button, on the left, that is necessary to start a sliding menu containing application options. Implemented function regards the details. This function is able to make clickable the imported elements and becomes necessary to retrieve the information of the selected family type from the DB. The visualization of parameters of each element takes place in a very dynamic way, showing a grid with name on the left and its relative value/formula on the right (Figure 7). This last field is editable to guarantee the user the possibility to 
modify it. When the screen is closed, the application connects to the DB and updates fields of the displayed table.

Revit database is updated using a specific Dynamo script that connects to MySQL database and downloads data related to a single element.

\section{CONCLUSIONS AND FUTURE LINES}

The proposed paper, thanks to the performed case study, is able to validate a workflow from the sematic model to the visualization in a VR and mobile environment (Figure 8).

The procedure here illustrated allows an interactive visualization and access to $3 \mathrm{D}$ models based on the architectural shapes from treatises using virtual reality (VR) techniques. The methodology developed and implemented is based on VR devices and integrated user interfaces with Unity. The obtained results allow us to access and view digital historical architecture objects semantically aware and with a parametric intelligence. Finally, the workflow is assessed interacting with them using an easy and intuitive interface. This application permits the user, once immersed into a virtual world, to explore a 3D scene and modify objects starting from editable parameters.

Moreover, this work shows that it is possible to create a semiautomatic system to transfer information into investigable databases that allow to re - create automatically fields and values related to 3D model. Another important aspect of this workflow is the possibility to interact directly with the 3D model, by the external viewer, through user-defined fields and not predefined ones obtained with management and creation software of the HBIM model.

It is also possible to generalize the pipeline and to stress the queries, in addition several developments and further advances are expected from the chance to have a return path. As a matter of fact, the illustrated procedure is valid both for a forward and a return workflow. This is not a mere scalability process, but a solid method for other types of data enrichment and data assessment.

In this way future works will enable to directly implement data in a remote and multidisciplinary path and in a non - desktop environment and provide data enrichment for HBIM. The HBIM model is dismantled into different elements and then studied as a project database for system programming and developments.

A major achievement from this research is to support a semantic management of 3D data relating to the knowledge of historical buildings and to develop an innovative application in the Cultural Heritage field. More in general, it enables the spreading of suitable HBIM families starting from historical analysis: the effectiveness of the work lies in adopting standard data management and allowing parametric model compliant with the state of art visualization and interoperable with VR representation for historical heritage.

\section{Semantic Modeling}

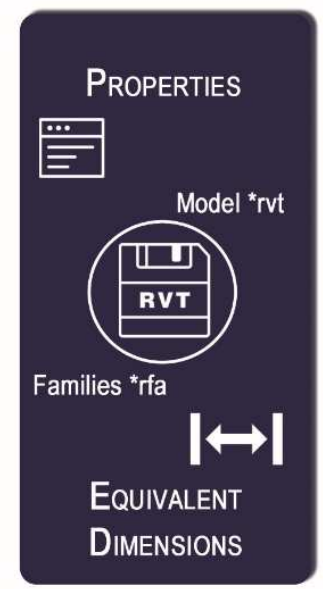

Parametric InTElLIGenCE

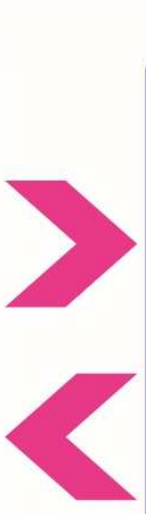

EXPORT/IMPORT

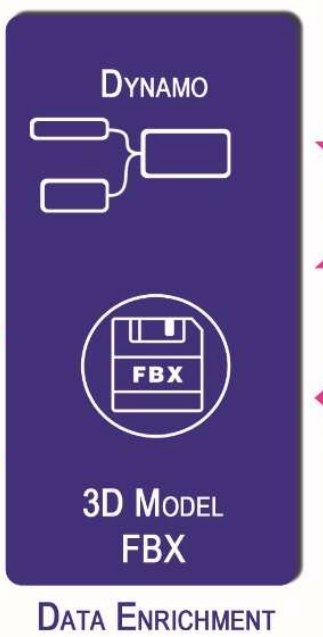

Data Managment

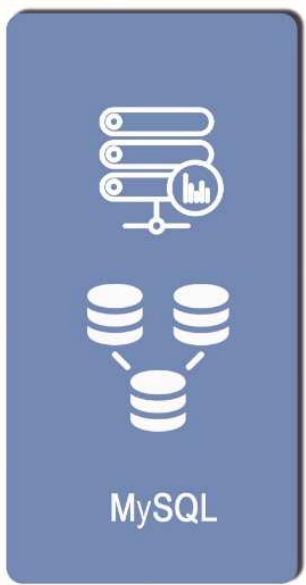

Aware 3D Database

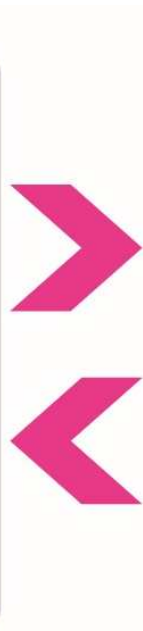

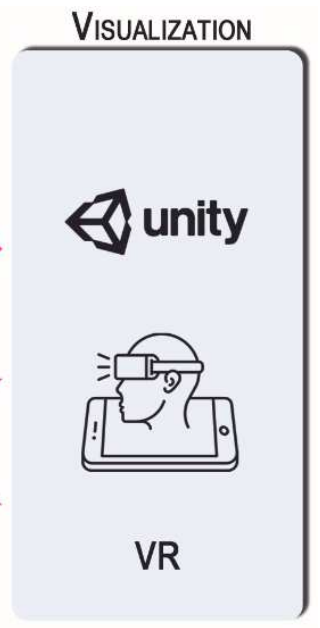

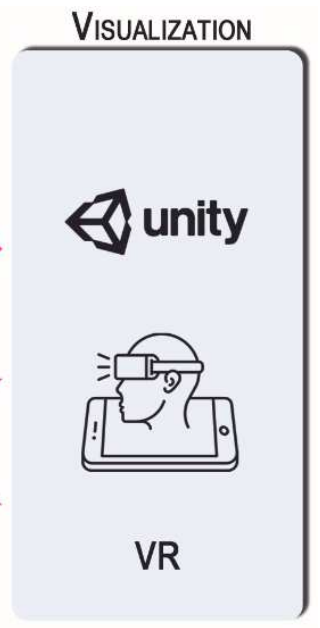

VR

PORTABILITY

Figure 8. Workflow of method: from semantic modeling to visualization through export and data management phase.

\section{REFERENCES}

Apollonio, F. I., Gaiani, M. and Sun, Z., 2013. '3D modeling and data enrichment in digital reconstruction of architectural heritage', ISPRS Archives, 5, pp. W2-W2.

Apollonio, F. I. and Giovannini, E. C., 2015. 'A paradata documentation methodology for the Uncertainty Visualization in digital reconstruction of $\mathrm{CH}$ artifacts', SCIRES-IT SCIentific RESearch and Information Technology, 5(1), pp. 1-24. doi: 10.2423/I22394303V5N1P1.

Bassier, M., Hadjidemetriou, G., Vergauwen, M., Van Roy, N. and Verstrynge, E., 2016. 'Implementation of scan-to-BIM and FEM for the documentation and analysis of heritage timber roof structures', in Lecture Notes in Computer Science (including subseries Lecture Notes in Artificial Intelligence and Lecture Notes in Bioinformatics). doi: 10.1007/978-3319-48496-9_7.

Battini, C. and Sorge, E., 2016. 'Dynamic Management of Survey data and Archaeological Excavation. The case study of the Amphitheatre of Volterra', SCIRES-IT - SCIentific RESearch and Information Technology, 6(2), pp. 119-132. doi: 10.2423/I22394303V6N2P119.

Battini, C. and Vecchiattini, R., 2017. 'Survey and restoration: New ways of interaction', in International Archives of the Photogrammetry, Remote Sensing and Spatial Information Sciences - ISPRS Archives, pp. 655-662. doi: 10.5194/isprsArchives-XLII-5-W1-655-2017.

Biagini, C., Capone, P., Donato, V. and Facchini, N., 2016. 'Towards the BIM implementation for historical building 
restoration sites', Automation in Construction. Elsevier, 71, pp. 74-86. doi: 10.1016/J.AUTCON.2016.03.003.

Bianchini, C., Inglese, C., Ippolito, A., Maiorino, D. and Senatore, L. J., 2016. 'Building Information Modeling (BIM): Great Misunderstanding or Potential', Handbook of Research on Emerging Technologies for Digital Preservation and Information Modeling. IGI Global, p. 67.

Chu, M., Matthews, J. and Love, P. E. D., 2018. 'Integrating mobile Building Information Modelling and Augmented Reality systems: An experimental study', Automation in Construction, 85, pp. 305-316. doi: 10.1016/j.autcon.2017.10.032

Clini, P., Quattrini, R., Frontoni, E., Pierdicca, R. and Nespeca, R., 2016. Real/not real: Pseudo-holography and augmented reality applications for cultural heritage, Handbook of Research on Emerging Technologies for Digital Preservation and Information Modeling. doi: 10.4018/978-1-5225-06805.ch009.

COMMITTE, U., 2017. 'UNI 11337-4:2017 Edilizia e opere di ingegneria civile - Gestione digitale dei processi informativi delle costruzioni - Parte 4: Evoluzione e sviluppo informativo di modelli, elaborati e oggetti', pp. 1-118.

Dalton, B., Parfitt, M., Du, J., Zou, Z., Shi, Y. and Zhao, D., 2018. 'Immersive Visualization of Building Information Models', Automation in Construction, 85 (August 2016), pp. 1-20. doi: 10.1016/j.autcon.2017.10.009.

Garagnani, S., 2015. Semantic representation of accurate surveys for the cultural heritage: BIM applied to the existing domain, Handbook of Research on Emerging Digital Tools for Architectural Surveying, Modeling, and Representation. doi: 10.4018/978-1-4666-8379-2.ch009.

Gros, P., 1997. Vitruvio. De Architectura. Edited by Einaudi.

Handel, O., Gümüş, E., Papoutsis, E. and Amann, J., 2009.
'Dynamic Visualization of Pedestrian Simulation Data', Proc. of the 27th Forum Bauinformatik, pp. 1-9.

Jiménez Fernández-Palacios, B., Morabito, D. and Remondino, F., 2017. 'Access to complex reality-based 3D models using virtual reality solutions', Journal of Cultural Heritage, 23, pp. 40-48. doi: 10.1016/j.culher.2016.09.003.

Liu, Y., Tanudjaja, G., Jiang, Z. and Beck, N., 2016. Workflow of Exporting Revit Models to Unity. Available at: https://bim.wikispaces.com/file/view/Revit_3DS_Unity+Wor kflow.pdf.

López, F. J., Lerones, P. M., Llamas, J., Gómez-GarcíaBermejo, J. and Zalama, E., 2017. 'A Framework for Using Point Cloud Data of Heritage Buildings Toward Geometry Modeling in A BIM Context: A Case Study on Santa Maria La Real De Mave Church', International Journal of Architectural Heritage. doi: 10.1080/15583058.2017.1325541.

De Luca, L., 2014. 'Methods, formalisms and tools for the semantic-based surveying and representation of architectural heritage', Applied Geomatics, 6(2), pp. 115-139. doi: 10.1007/s12518-011-0076-7.

Morolli, G., 1986 Le membra degli ornamenti. Sussidiario illustrato degli ordini architettonici. Edited by Alinea.

Murphy, M., McGovern, E. and Pavia, S., 2009. 'Historic building information modelling (HBIM)', Structural Survey. Emerald Group Publishing Limited, 27(4), pp. 311-327.

Santagati, C. and Lo Turco, M., 2016. 'From structure from motion to historical building information modeling: populating a semantic-aware library of architectural elements', Journal of Electronic Imaging. International Society for Optics and Photonics, 26(1), p. 11007. doi: 10.1117/1.JEI.26.1.011007. 